\title{
ShopTalk: Independent Blind Shopping Through Verbal Route Directions and Barcode Scans
}

\author{
John Nicholson ${ }^{*}$, , Vladimir Kulyukin ${ }^{*, 1}$ and Daniel Coster $^{2}$ \\ ${ }^{1}$ Department of Computer Science, Utah State University, Logan, UT 84322-4205, USA \\ ${ }^{2}$ Department of Mathematics and Statistics, Utah State University, Logan, UT 84322-4205, USA
}

\begin{abstract}
Independent shopping in modern grocery stores that carry thousands of products is a great challenge for people with visual impairments. ShopTalk is a proof-of-concept wearable system designed to assist visually impaired shoppers with finding shelved products in grocery stores. Using synthetic verbal route directions and descriptions of the store layout, ShopTalk leverages the everyday orientation and mobility skills of independent visually impaired travelers to direct them to aisles with target products. Inside aisles, an off-the-shelf barcode scanner is used in conjunction with a software data structure, called a barcode connectivity matrix, to locate target product on shelves. Two experiments were performed at a real world supermarket. A successful earlier single-subject experiment is summarized and a new experiment involving ten visually impaired participants is presented. In both experiments, ShopTalk was successfully used to guide visually impaired shoppers to multiple products located in aisles on shelves. ShopTalk is a feasible system for guiding visually impaired shoppers who are skilled, independent travelers. Its design does not require any hardware instrumentation of the store and leads to low installation and maintenance costs.
\end{abstract}

\section{INTRODUCTION}

Different environments present different challenges to people with visual impairments. Some environments are challenging due to their large size or lack of structure, whereas other environments, although structurally regular, are functionally challenging due to the complexities of the tasks that must be performed in them. The list of the most functionally challenging environments for individuals with visual impairments is topped by shopping complexes [1]. This difficulty can be understood given that a typical modern supermarket stocks an average of 45,000 products and has a median store size of 4,529 square meters (48,750 square feet) [2].

Many people with visual impairments do not shop independently. They receive assistance from a friend, a relative, an agency volunteer, or a store employee [3]. Depending on the assistant's availability, the shopper may need to postpone the shopping trip. A completely blind participant in the experiment reported later in this article related a personal anecdote in which he was required to wait for over 15 minutes before a store employee was able to assist him. Although he is a highly skilled independent traveler, capable of independently walking around Logan, UT, and the Utah State University (USU) campus, he is unable to shop independently. His account agrees with some stories in popular press on the shopping experiences of individuals with visual impairments [4].

Service delays are not the only problem that visually impaired shoppers face in modern supermarkets. In March

*Address correspondence to these authors at the Department of Computer Science, Utah State University, Logan, UT 84322-4205, USA; Fax: 1-435797-3265;

E-mails: john.nicholson@aggiemail.usu.edu, vladimir.kulyukin@usu.edu
2007, the first two authors conducted an informal focus group as part of a regular monthly meeting of the Logan Chapter of the National Federation of the Blind. The group consisted of six visually impaired individuals from Cache Valley, Utah who were independent travelers, held part-time or full-time jobs, used public transportation, and walked independently around their neighborhoods. In addition to service delays, they reported other difficulties, such as staffers not familiar with the store layout and assistants who became irritated with long searches. One participant said that on several occasions she was assigned staffers who could not speak English and could not read the products' ingredients to her. The consequences of these were that the participants either had to give up searching for the product that they wanted or had to settle for products that were distant substitutes.

Another grocery shopping option for people with visual impairments is to use a home delivery service, such as the Internet-based PeaPod [5] or those provided by some brickand-mortar grocery stores. Shopping lists are usually provided over the phone or through online websites. The two main disadvantages of delivery services are that they are not universally available and that they require the shopper to schedule and wait for the delivery. Although these services are useful, personal independence is reduced and spontaneous shopping is not possible in this context.

In a previous investigation of independent blind shopping, we distinguished two types of grocery shopping: smallscale and large-scale [6]. In small-scale shopping, the shopper buys only a few items that can be carried by hand or in a hand basket. Large-scale shopping involves buying more products and will typically necessitate the use of a shopping cart. In another investigation, we used the ergonomics-forone framework [7] to interview ten people with visual impairments about their grocery shopping experiences $[8,9]$. The subsequent analysis of the interviews identified five 
subtasks of the generic grocery-shopping task: 1) traveling to the store; 2) finding the needed grocery items in the store; 3 ) getting through a cash register; 4) leaving the store; and 5) getting home.

This article focuses on the second task of finding the required grocery items in the store. We confine the scope of our investigation to shopping for items stocked on the aisle shelves in a typical supermarket. We further restrict this investigation to small-scale shopping. Shopping for frozen products and produce as well as large-scale shopping are beyond the scope of this investigation.

This article is organized as follows. We first perform a functional analysis of the generic grocery-shopping task and propose a trichotomous spatial ontology for grocery stores. We then describe ShopTalk and discuss how its design and implementation were informed by our analysis of the grocery shopping task and the focus group on independent blind shopping. Next, we present a study of 10 participants with visual impairments using ShopTalk in Lee's Marketplace, a supermarket in Logan, UT. Finally, we review related work and present our conclusions.

\section{GROCERY SHOPPING TASK AND SPATIAL TRICHOTOMY}

The task of searching for grocery items, when performed by a typical sighted shopper with a shopping list, has three stages. The first stage begins after the shopper has entered the store and possibly obtained a hand basket or a cart. The shopper orients herself to the store layout and begins to search for the first item on her list. We refer to the current item on the list as the target product. During the second stage, the shopper walks around the store trying to localize the target product. When the item is found, the shopper places it into her basket and proceeds to search for the next item on the list. The second stage is completed when the last item on the list is found. In the third and final stage, the shopper travels from the last item's location to the cashier, pays for her items, and leaves the store.

The second stage requires that the shopper both locate and identify each individual product on her shopping list. Product location involves determining where a product is located in the store and then navigating to that location. Product identification is the process of ensuring that the found product is the target product. Product identification may be necessary, because the target product may not be at its expected location. The product may be sold out or a different item may be in the target product's location. Product identification is also critical for distinguishing between certain types of products. For example, many canned vegetables can only be differentiated from one another after performing a visual inspection of their label.

When the shopper is looking for products, she is moving through the space within the grocery store. Inspired by Barbara Tversky's research [10], we categorized different types of space within the shopping task in order to better understand the task. In our previous research with RoboCart [6, 9], a robotic shopping cart for the shoppers with a visual impairment, we used the standard spatial dichotomy (locomotor $v s$ haptic) from the blind navigation literature [11]. The locomotor space includes areas of large-scale movement around the grocery store. The haptic space is the space in the immediate vicinity of the shopper's body. RoboCart guides the shopper around the grocery store in the locomotor space. When the robot reaches the approximate area of the desired product, the shopper uses a bar code scanner to search for the product. This search involves the shopper reaching out to products in the haptic space.

In this article, we extend the dichotomy of locomotor and haptic spaces with the category of search space to better represent the task of searching for individual products in aisles. The search space is defined as a small space around the shopper where the shopper performs limited locomotion, narrowing in on the specific location of the target product. Because there is locomotion, the search space overlaps with the locomotor spaces. Our trichotomy may be appropriate for both sighted shoppers and visually impaired shoppers, and draws on the research by Freundschuh and Egenhofer (FE) [11] who provide a comprehensive review of the previous work on categorization of space.

The task of locating a product in the store can be viewed as a process of the shopper moving through the three spaces. In the locomotor space, the shopper travels from her current location to the general area of the target product. This involves such actions as walking to the correct aisle, entering an aisle, and walking to the area where the shopper expects the product to be. Moving to the product's expected area does not guarantee that the shopper will be directly in front of the product. Although stores tend to group similar products together in sections, some of the product sections, e.g. canned soups or pasta sections, can be too large for the shopper at one end of such a section to physically reach the products at the other end of that section without locomotion.

Once in the vicinity of the product, the shopper shifts from the locomotor space to the search space. In the search space, the amount of locomotion required may be as small as a couple of steps before the shopper can place herself directly within reach of the target product. At other times, such as when searching for a product in large sections of similar products, the amount of locomotion may be a few meters, but will be still small in comparison to the amount of locomotion required in the locomotor space. One feature of the search space which is different from the locomotor space is that a small amount of visual or haptic scanning may be required to determine the position of the target product.

When the target product is within the reach of the shopper, the product is considered to be in the shopper's haptic space. This space requires no locomotion on the part of the shopper, because the shopper can now physically grasp the target product.

\section{PRODUCT LOCATION ASSISTANCE FOR THE VISUALLY IMPAIRED}

The above functional analysis of the shopping task sheds light on the requirements for independent shopping solutions for the people with visual impairments. To guarantee independence, any device must, at the very least, enable the shopper to navigate the store reliably and to search for and retrieve individual products. One way to satisfy these requirements is to instrument the store with various sensors, e.g., Talking Lights [12] or RFID tags [13], and give the shopper a signal receiver that provides directions as a function of the identities of the sensors detected in the environ- 
ment. The same instrumentation approach can be carried over to the task of product search and retrieval. For example, one can assume that every item in the store is instrumented with a sensor, e.g. an RFID tag that can be used for product search and identification.

Since the initial cost of instrumentation and subsequent maintenance are two important factors that often prevent the adoption of assistive technologies, ShopTalk's design makes a commitment to zero additional hardware instrumentation beyond what is already installed in a typical supermarket. ShopTalk is built on the explicit assumption that simple verbal route directions and layout descriptions can be used to leverage the everyday O\&M skills of independent travelers to successfully navigate in the store.

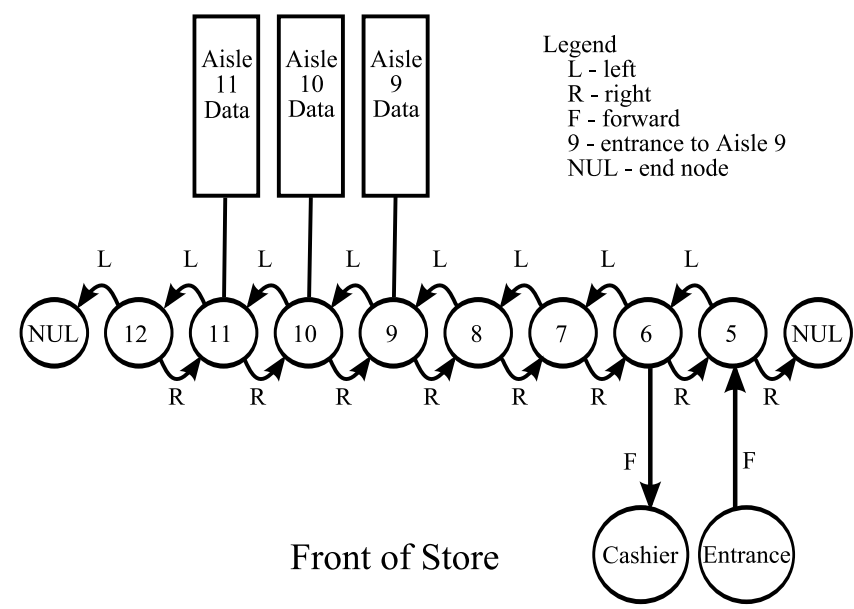

Fig. (1). Topological map representation of the store used in the experiments.

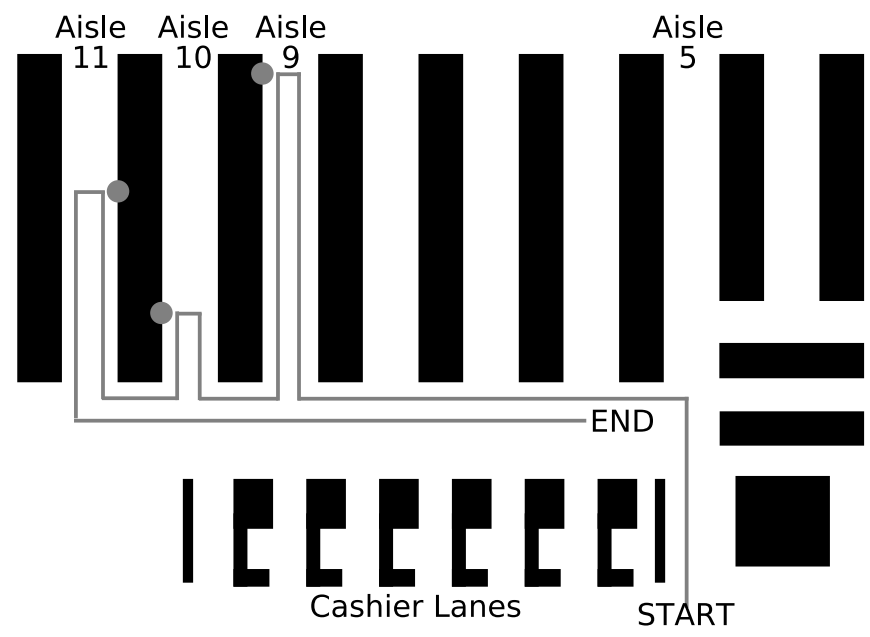

Fig. (2). Route map of the store used in the experiments. Dots represent the approximate locations of target products.

In ShopTalk, the environment is represented in two data structures. The first data structure is a topological map of the locomotor space. ShopTalk's topological map is given in Fig. (1) with a general map of the store in Fig. (2). The topological map is a directed graph whose nodes are decision points: the store entrance, aisle entrances, and cashier lane entrances. Other decision points can be added as needed. The edges are labeled with directions. Due to the regularity of modern supermarkets and the constraints of our problem (small-scale shopping for items stocked on aisle shelves), we found it sufficient to have three directional labels: left, right, and forward. The topological map is the only software instrumentation requirement for ShopTalk to become operational. The map is built at installation time by walking through the store, noting decision points of interest, and then representing them in the map.

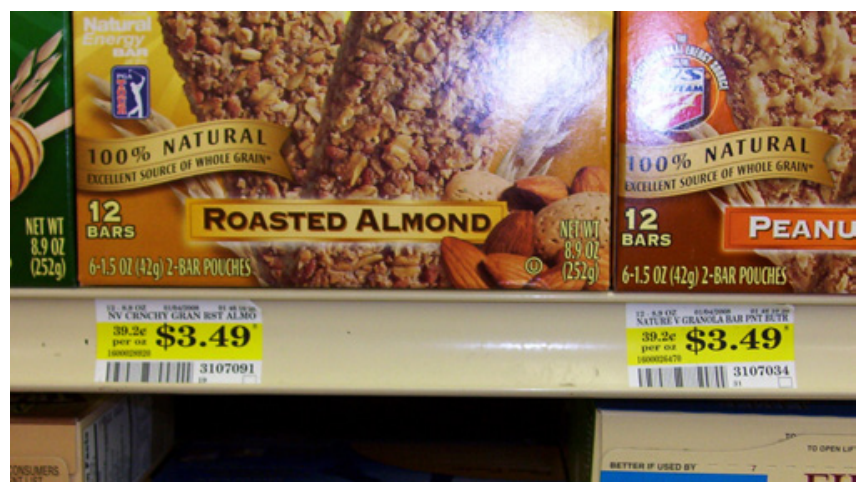

Fig. (3). Two shelf barcodes with product names and price information.

The second data structure is designed to take advantage of the inventory systems already used by many large- and medium-sized grocery stores. These inventory systems place barcodes on the shelves immediately beneath every product as seen in Fig. (3). Shelf barcodes assist the store personnel in managing the product inventory. When the locations of all shelf barcodes in the store are known, this information can be used to guide the shopper through the haptic and search spaces to the most probable location of the target product on the shelf because, under most circumstances, products are located directly above their corresponding shelf barcodes.

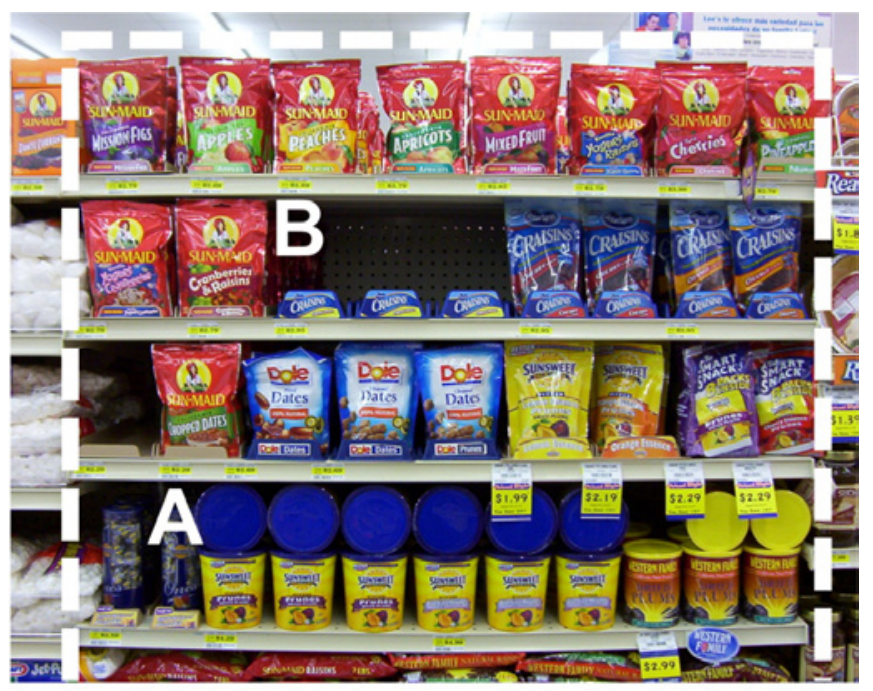

Fig. (4). Top half of a shelf section. Shelf section edges are marked by the dotted line. Product A is on shelf 3 in position 2. Product B is on shelf 1 in position 3 .

The second data structure is the barcode connectivity matrix (BCM). Each shelf barcode is associated with several types of information (see Fig. (4) and Table 1). First, a barcode is located in a specific aisle. Second, the barcode is on either the left or the right side of that aisle. Third, the barcode is in a specific shelf section. A shelf section is a group of shelves approximately 1.22 meters ( 4 feet) wide and includes the shelves from the top shelf to the bottom shelf. 
Shelf sections are numbered so that shelf section 1 is the section closest to the storefront. As one moves to the back of the store, shelf section numbers increase. Fourth, the barcode resides on a specific shelf in a shelf section. The top shelf in a shelf section is always labeled as shelf 1 , with the shelves below increasing in number. Finally, the barcode's relative position on the shelf is recorded. This position is not a $2 \mathrm{D}$ coordinate measured in some distance units, but a relative position based on how many products are on the same shelf.

Table 1. Entries in the Barcode Connectivity Matrix, Specifically Items $A$ and $B$ in Fig. (4)

\begin{tabular}{|c|c|c|}
\hline & Product A & Product B \\
\hline \hline Aisle & 9 & 9 \\
\hline Aisle Side & Left & Left \\
\hline Section & 16 & 16 \\
\hline Shelf & 3 & 1 \\
\hline Position & 2 & 3 \\
\hline Barcode & 3340726 & 3343035 \\
\hline Description & Chopped Dates & California Peaches \\
\hline
\end{tabular}

The BCM can be built automatically from the store's inventory database. However, we were not granted access to the inventory management system of Lee's Marketplace, because it is the supermarket's policy not to give access to their pricing information to third parties. The management told us, in generic terms, what information their inventory database contains and allowed us to scan all shelf barcodes in three (aisles 9, 10, and 11) of the twelve grocery aisles. We developed a simple graphical user interface (GUI) for entering the necessary information associated with a specific barcode and proceeded to scan all items in three aisles. It took us 40 hours to scan and manually enter all the information for the 4,297 products found in the three aisles. It should be noted again that the GUI development and the manual entry of product information was done exclusively for research purposes, because we were not granted permission to the store's inventory management system. These efforts will be unnecessary in the production version of the system owned by the store in which the BCM is computed automatically from the store's inventory database.

\section{Hardware}

ShopTalk's hardware (see Fig. 5) consists of a computational unit, currently an OQO model 01 , a Belkin numeric keypad, a Hand Held Products IT4600 SR wireless barcode scanner and its base station, and a USB hub that connects all components. To help carry the equipment, the user wears a small CamelBak backpack. The numeric keypad is attached by velcro to one of the backpack's shoulder straps, and to ensure adequate air circulation, the OQO is placed in a wire frame attached to the outside of the backpack. The remaining components - the barcode scanner's base station, the USB hub, and all connecting cables - are placed inside the backpack's pouch. Since the system gives speech-based instructions to the user, the system has a small headphone.

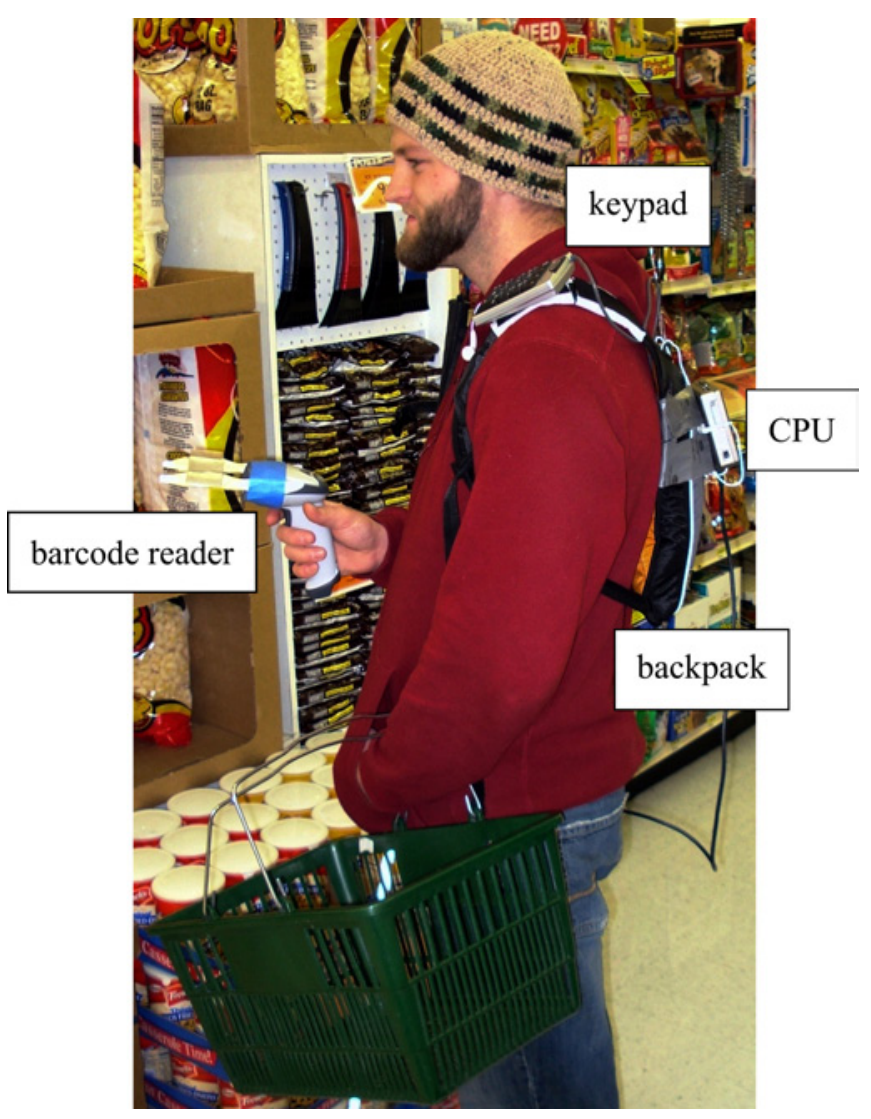

Fig. (5). ShopTalk's hardware.

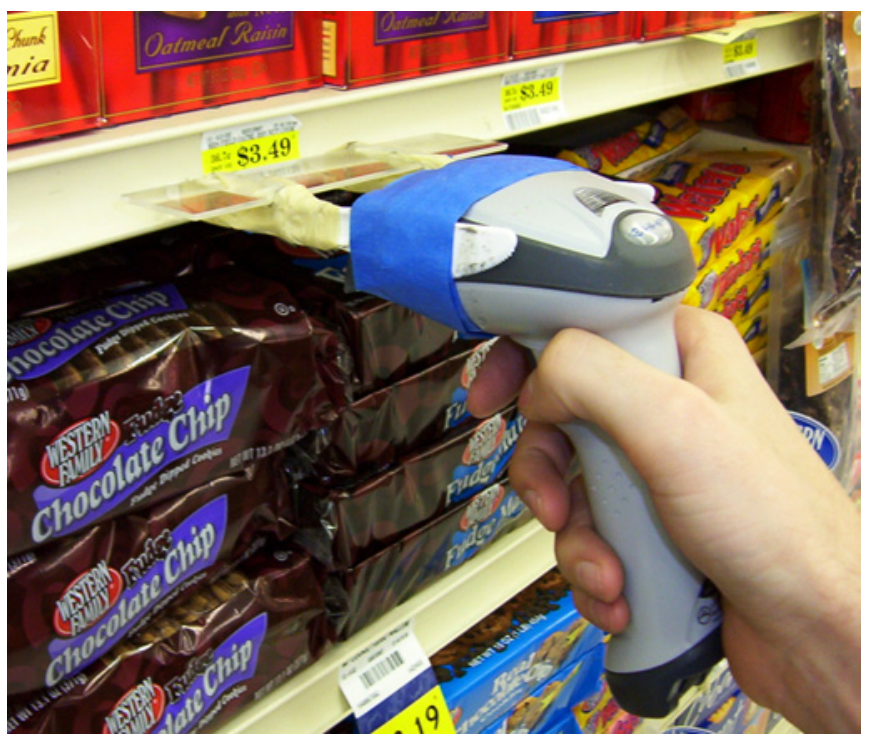

Fig. (6). The barcode scanner, with the added stabilizers, resting of a shelf lip.

The barcode scanner is placed in a shopping basket carried by the shopper and is retrieved when needed. The scanner was slightly modified (see Fig. 6) to take advantage of the fact that many supermarkets have shelves that curl down and have a small lip at the bottom. We attached plastic stabilizers to the front of the scanner that rest on the shelf lips when a shopper is scanning barcodes. The stabilizers make it easier for the shopper to align the scanner with shelf barcodes thereby reducing the time to achieve a successful scan. 
The components listed above constitute but one possible hardware realization of a shopping aid. The present realization was a direct consequence of budgetary constraints and equipment at hand. The hardware can be further miniaturized. For example, the software could be installed on a mobile smartphone allowing the phone to act as the computational unit. Our current barcode scanner could also be replaced with a smaller model. Finding an optimal hardware configuration is an ergonomic problem that we plan to address in our future research. Our primary objective in this investigation was to test the validity of our hypothesis that verbal route descriptions and barcode scans are sufficient for independent shopping for shelved items in a supermarket.

\section{Verbal Route Directions}

When guiding the shopper in the locomotor space, ShopTalk issues route instructions in two modes: location unaware mode (LUM) and location aware mode (LAM). The LUM is used in the locomotor space, whereas the LAM is reserved for the search and haptic spaces. The LUM instructions are generated from the topological map of the store and a database of parameterized route directions. A parameterized route direction is an expression, such as Turn $X$ or You are at $X$ where $X$ can be replaced with a context-sensitive word or phrase.

Given the start and end nodes of a path, the actual route directions are constructed from the database of parameterized route directions by replacing the parameters with appropriate fillers from the topological map. The following illustrates how a shopper interacts with the LUM instructions. These instructions would be generated if the shopper was in the middle of aisle 9 and needed to be guided to the entrance of aisle 11 .

1. Shopper presses the keypad's Enter key to request the next instruction.

2. ShopTalk gives the instruction: "Turn right. Walk forward to the entrance of the aisle."

3. Shopper reaches the aisle entrance and presses Enter again.

4. ShopTalk gives the instruction: "Turn right. You are at entrance to aisle 9. Walk forward until you detect the entrance to aisle 11."

This mode is location-unaware because the system itself is unaware of the shopper's actual location and orientation. Since there are no external sensors for detecting the shopper's current location, ShopTalk explicitly relies on the shopper's orientation and mobility skills. It assumes that the shopper can detect the environmental cues needed to make sense of the provided verbal instructions and localize herself in the environment. For example, in the above example, the first instruction assumes that the shopper is facing the shelf after retrieving a product and putting it into her basket. If the shopper is already facing the entrance of the aisle when the instruction is given, the first turn instruction can be ignored by the shopper. As one participant put it to us in an informal conversation, "I use only those instructions that make sense to me at the moment and ignore the rest." That is the point of ShopTalk: it is always the shopper who makes the final decision about what action to execute.
ShopTalk's LUM is conceptually no different from being guided on a cell phone by a fellow shopper who knows the store. There is some research evidence $[14,15]$ that people with visual impairments share route descriptions and guide each other over cell phones. The most intriguing aspect of this information sharing is that the guide is not present en route and the guidee must make sense of the guide's instructions independently. Prior to testing ShopTalk, we hypothesized that, over time, as the shopper uses the system repeatedly in the same store, the shopper would internalize the store layout and would not need the LUM instructions in the locomotor space at all. Therefore, the LUM instructions are given to the shopper only when the shopper requests them by pressing the Enter key. If the routes and the store layout have been internalized, the shopper can choose not to request the LUM instructions.

The LUM guidance may lead to occasional navigation problems. The shopper may miscount waypoints, be blocked by displays or other shoppers, or have to deal with unforeseen events, such as employees restocking the shelves, all of which could result in temporary disorientation. Of course, travelers with visual impairments routinely face these problems while interpreting someone else's verbal route directions on the street. The key difference is that with ShopTalk the shopper can immediately determine her present location so long as she can scan a barcode in any aisle.

A barcode scan switches the mode of instruction from LUM to LAM. As soon as a barcode is scanned, the exact location of the shopper is known to the system and the system issues location-aware instructions on how to get to the target product. If the shopper becomes lost in the locomotor space, a barcode scan will inform her about her present location. Thus, barcode scans function not just as product identification cues in the search and haptic spaces but also as error correction cues in the locomotor space. In the search space, barcode scans index into the BCM enabling the system to guide the shopper gradually through the search space to the haptic space. In the haptic space, the process of scanning products guides the shopper to the exact location of the target product on the shelf.

When the last product on the shopping list is located, the system gives the shopper verbal instructions on how to get to the cashier. This location is somewhat easier to find because there are specific types of noises associated with the cashier area that assist the shopper in finding it.

\section{METHODS}

In this section, we report on two sets of experiments we have used to evaluate ShopTalk's feasibility and effectiveness. We begin by summarizing a single subject pilot study and then proceed to give a detailed account of a multiple participant study. In both studies, ShopTalk was evaluated as an aid for a small-scale shopping task. Each experiment only required the participants to shop for three products at a time.

\section{Single Participant Pilot Study}

We first tested ShopTalk in a pilot study with one visually impaired participant to determine whether such a system was feasible. The details of our pilot study are reported in [16]. Here we briefly summarize the main findings, because 
it informed the subsequent design and execution of the multi-participant experiment.

We formulated three hypotheses for our single subject study. First, a blind shopper with independent O\&M skills can successfully navigate the supermarket using only verbal directions. Second, verbal instructions based on run time barcode scans are sufficient for target product localization in an aisle. Third, as the shopper repeatedly performs the shopping task, the total traveled distance approaches an asymptote.

To test these hypotheses, we built the BCM for one aisle in Lee's Marketplace, a local supermarket in Logan, UT. Several product sets were selected from that aisle. A product set was a set of three randomly chosen products. Each product set had one item randomly chosen from the aisle's front, middle, and back. Three product sets contained items only from the aisle's left side, three sets contained items only from the aisle's right side, and one contained two items from the left side and one from the right. To make the shopping task realistic, each product set contained one product from the top shelf, one product from the bottom shelf, and one product from a middle shelf.

The participant was an independent blind (only light perception) guide dog handler in his mid-twenties. In a 10minute training session before the first run, the basic concepts underlying ShopTalk were explained to him to his satisfaction. A run consisted of the participant starting at the entrance of the store, traveling to the target aisle (aisle 9), locating the three products in the current product set, and, after retrieving the last product in the set, traveling to a designated cashier.

Sixteen runs were completed with at least one run for each product set in five one-hour sessions at Lee's Marketplace. All three of our hypotheses appeared to be reasonable for this participant. First, the participant was able to navigate to the designated aisle using only ShopTalk's verbal route directions. Second, using only ShopTalk's verbal search instructions based on the BCM and barcode scans, the participant was able to find all products for all 16 runs. Third, the participant's overall navigation distance decreased with each subsequent run for the same product set.

\section{Multiple Participant Study}

After completing the single participant study, we conducted a similar study with 10 participants with a visual impairment. The participants were recruited by referral from the greater Logan area of Utah with the help of the Logan, Utah Chapter of the National Federation of the Blind. All participants had O\&M training. Table 2 gives the collected demographic data on the participants. Each participant was paid a $\$ 45$ honorarium.

In our second study, the locations of shelf barcodes were recorded for three aisles $(9,10$, and 11) in Lee's Marketplace. Table 3 shows how many items were scanned in the three aisles to build the BCM. While scanning the barcodes, the names of 694 products were recorded. From these named products, one product was randomly chosen from each aisle for a total of three products. These three products represented the product set for this experiment.
Table 2. Participants' Demographic Data for the Multiple Participant Study. Participants Who Listed Both as Their Usual Aid Did Not Indicate a Preference for Using a Cane More or Less Often Than a Guide Dog

\begin{tabular}{|c|c|c|c|c|c|c|}
\hline ID & Gender & Age & $\begin{array}{c}\text { Vision } \\
\text { Level }\end{array}$ & $\begin{array}{c}\text { Usual } \\
\text { Aid }\end{array}$ & $\begin{array}{c}\text { Experiment } \\
\text { Aid }\end{array}$ & $\begin{array}{c}\text { Other } \\
\text { Disability }\end{array}$ \\
\hline \hline 1 & female & 31 & low & cane & cane & yes \\
\hline 2 & male & 24 & none & dog & dog & no \\
\hline 3 & female & 20 & none & both & cane & no \\
\hline 4 & male & 18 & low & cane & cane & no \\
\hline 5 & male & 29 & low & dog & dog & no \\
\hline 6 & male & 23 & none & both & cane & no \\
\hline 7 & male & 24 & low & cane & cane & no \\
\hline 8 & female & 26 & low & cane & cane & no \\
\hline 9 & male & 22 & low & dog & dog & no \\
\hline 10 & female & 18 & low & none & none & \\
\hline
\end{tabular}

Table 3. The Number of Items Scanned in Three Aisles for the Experiment

\begin{tabular}{|c|c|c|}
\hline Aisle & Number of Barcodes & Names Recorded \\
\hline \hline 9 & 1569 & 197 \\
\hline 10 & 1073 & 200 \\
\hline 11 & 1655 & 297 \\
\hline Total & 4297 & 694 \\
\hline
\end{tabular}

As is often the case with studies involving participants with visual impairments, it is not feasible to test, in a statistically significant way, all contributing factors in a single study due to the uneven distribution of the population in the U.S. of those with visual impairments, with the majority living in just a few urban areas. Therefore, our hypotheses below address only a fraction of the factors outlined in the previous section.

- Hypothesis $1\left(\mathrm{H}_{1}\right)$ : Using only verbal route directions, a person with visual impairments can successfully navigate the locomotor space in a grocery store.

- Hypotheses $2\left(\mathrm{H}_{2}\right)$ : Verbal instructions based on barcode scans and the BCM are sufficient to guide shoppers with visual impairments to target products in the search and haptic spaces.

- Hypothesis $3\left(\mathrm{H}_{3}\right)$ : As participants repeatedly perform a shopping task, the total distance they travel approaches the distance traveled by a blind shopper being guided by a sighted person.

- Hypothesis $4\left(\mathrm{H}_{4}\right)$ : As participants repeatedly perform a shopping task, the total time taken to find products approaches the time needed by a blind shopper being guided by a sighted person. 
- Hypothesis $5\left(\mathrm{H}_{5}\right)$ : As participants repeatedly perform a shopping task, the number of barcode scans needed to find a target product decreases.

The experiment was performed during the grocery store's normal business hours. To minimize impact on the store staff and customers, experiments began at 9:00 PM and ended between 10:30 PM and 11:30 PM, depending on the participant's performance. Participants were given a one hour training session during which the system, the guidance techniques used, and the basic store layout were explained to them.

After the training session, each participant was led to the front of the store near its entrance and was given a shopping basket to carry. The participant was then asked to perform five runs of the experiment's shopping route. The route (see Fig. 2) began at the entrance of the store, went to each of the three products, and ended at the entrance of the cashier lane. Participants were not informed before starting the runs for which products they were going to shop.

During each run, participants were accompanied by two assistants. The first assistant monitored the participant's safety and recorded observations. The second assistant followed the participant with a Lufkin measuring wheel to measure the exact distance walked by the participant. The participant was asked to press Enter on the numeric keypad at the following times: when starting a run, when ready for the next locomotor instruction, after placing a found product in the shopping basket, and when reaching the entrance to the cashier lane. The system also recorded the time and barcode number whenever the participant scanned any barcode.

When a participant found the correct barcode for a product, the participant would pick up the product immediately above the barcode and place it in the shopping basket. This run was repeated five times for each participant. After each run, the participant would return to the store entrance to start the next run. All participants shopped for the same products in the same order and the same number of times.

\section{RESULTS}

Repeated measures analysis of variance (ANOVA) models were fitted to the data using the SAS system. Independent variables were gender, age, vision level (low or none), O\&M training (yes or no), usual navigation aid (cane, dog, or none), aid in experiment (cane, dog, or none), other navigation disabilities (yes or no), education level, and selfreported navigation skill level. Replication was achieved with 10 participants, and each participant made 5 runs, providing a repeated measures factor "runs" with 5 levels as the within-subjects factor. When participants reported their vision level, if they reported any level of vision they were recorded as low; only those reporting complete vision loss were recorded as none. The dependent variables were time and distance. The time variable included the time taken by the participant to reach the required aisles and the product search time taken to find the target products.

The overall success rate of product retrieval was $100 \%$. All ten participants were able to find all three products in every run. Verbal route instructions and barcode scans appeared to be sufficient to navigate the store and retrieve target products in grocery aisles. Thus, the null hypotheses as- sociated with research hypotheses $\mathrm{H}_{1}$ and $\mathrm{H}_{2}$ were rejected for our sample, and experimental evidence indicates that both $\mathrm{H}_{1}$ and $\mathrm{H}_{2}$ hold.

To test hypotheses $\mathrm{H}_{3}$ and $\mathrm{H}_{4}$, a baseline run was obtained by having a sighted person guiding a completely blind guide dog handler on a shopping run. The guide knew the store well and led the shopper to the same products in the same order, as was done during the experiment with the other participants. During the baseline run, the participant's guide dog followed the sighted guide. When a product was reached, the sighted guide would place the product in the basket the participant was carrying. The baseline run was performed once, took 133 seconds to complete, and had a distance of 117 meters (384 feet).

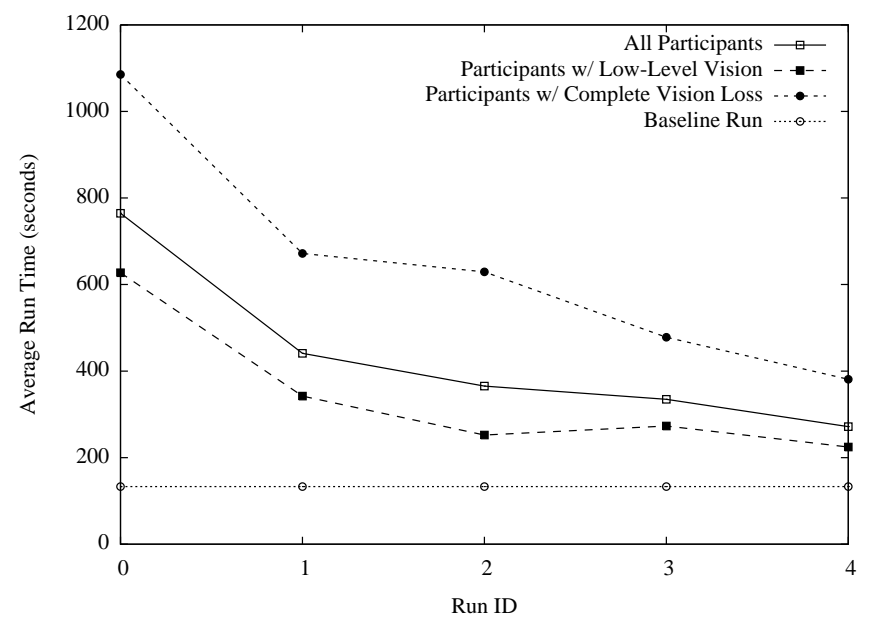

Fig. (7). The average run time over repeated runs. The baseline run's time is shown for comparison.

Fig. (7) shows that the average total time for run completion exhibited a downward trend over repeated runs. The decrease in run time across all 5 runs was found to be significant with $\mathrm{F}(4,9)=31.79, \mathrm{p}<0.0001$. Pairwise run time mean differences show that run 1 took significantly longer than the other runs. Run 2 took significantly longer than runs 4 and 5 , but not run 3 . Run 3 took significantly longer than run 5 but not run 4 . Runs 4 and 5 effectively had the same average time.

The participants' average run time, averaged over all 5 runs, differ significantly among the 10 participants with $\mathrm{F}(9,36)=22.62, \mathrm{p}<0.0001$. This is not surprising, given that our participants ranged from those with complete blindness who required a cane or guide dog to one participant who had enough vision to navigate without an aid. Thus, there appears to be sufficient evidence in our data to reject the null hypothesis associated with research hypotheses $\mathrm{H}_{3}$ and $\mathrm{H}_{4}$.

Further analysis was performed using repeated measures ANOVA to test several post hoc hypotheses comparing the total run time against several of the demographic factors. The model fitted in every case was a repeated measures model with between subjects factors selected from the list above: vision level, gender, age, and so on. Each of the 10 participants performed the run 5 times, thus there are 50 observations and 50 total degrees of freedom in the analysis. No significant effect was seen when looking at gender, age, O\&M training, primary navigational aid, education level, or the self-reported navigational skill level. Level of blindness 
did have an effect on the run time, which was expected. When the total run time was averaged over all five runs, the average time for the completely blind, 590.5 seconds, and the low level, 280.8 seconds, differed significantly with $\mathrm{F}(1,8)=21.45, \mathrm{p}=0.0017$. When the run number was considered, the effect of level of blindness is still significant but less so with $F(1,9)=65.83, p<0.0001$. The reason for the lower amount of significance was due to the longer times taken by the completely blind participants on runs 1 and 2 . It appears that over time the completely blind participants were able to increase their efficiency and began to approach the performance levels of the low vision participants.

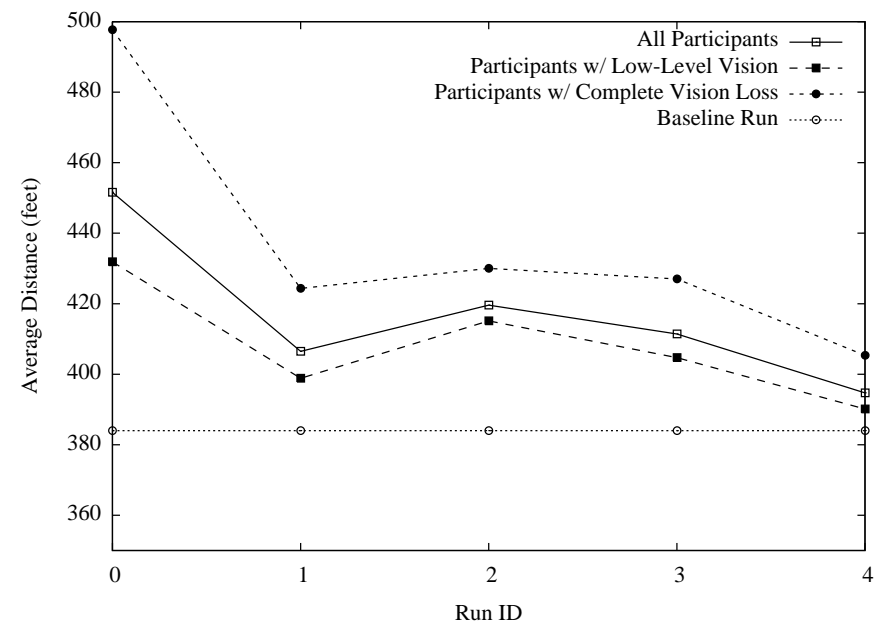

Fig. (8). The average distance walked for an entire run over repeated runs. The baseline run's distance is shown for comparison.

Like the average total time, the average distance traveled during a run fell as the runs were repeated (see Fig. 8). The analysis of the average distance is analogous to the analysis of the average total time. Effects of the run number and level of blindness were significant, with $\mathrm{F}(4,9)=5.52, \mathrm{P}=0.0159$ and $F(1,9)=53.13, p<0.0001$, respectively. The total distance decreased significantly with run number, and the total distance was significantly greater for the completely blind participants. This analysis indicates that, as the participants gained experience, their routes contained smaller errors related to distance. As discussed later in the Qualitative Observations section, it appeared that some of the increased accuracy came from learning the location of landmarks, which helped the participants to make better distance judgments.

Fig. (9) shows that the average number of products scanned per run also fell over repeated runs. The decrease in the number of products scanned across all 5 runs was found to be significant with $\chi^{2}(4)=24.26, \mathrm{p}<0.0001$ allowing the null hypothesis associated with $\mathrm{H}_{5}$ to be rejected. This appears to indicate that as the shoppers gained more experience, they became more efficient with the scanning process. The number of products scanned in the first two runs, when averaged over all 10 participants, did not differ significantly, but the average number of products scanned on runs 3,4 , and 5 were all significantly lower than run $1, \chi^{2}(1)=7.28$, $\mathrm{P}=0.0070, \chi^{2}(1)=13.23, \mathrm{P}=0.0003, \chi^{2}(1)=16.12, \mathrm{p}<$ 0.0001 . This suggests that by runs 3 or 4 the participants were approaching the asymptotic limit for the number of products they would need to scan on this particular route.

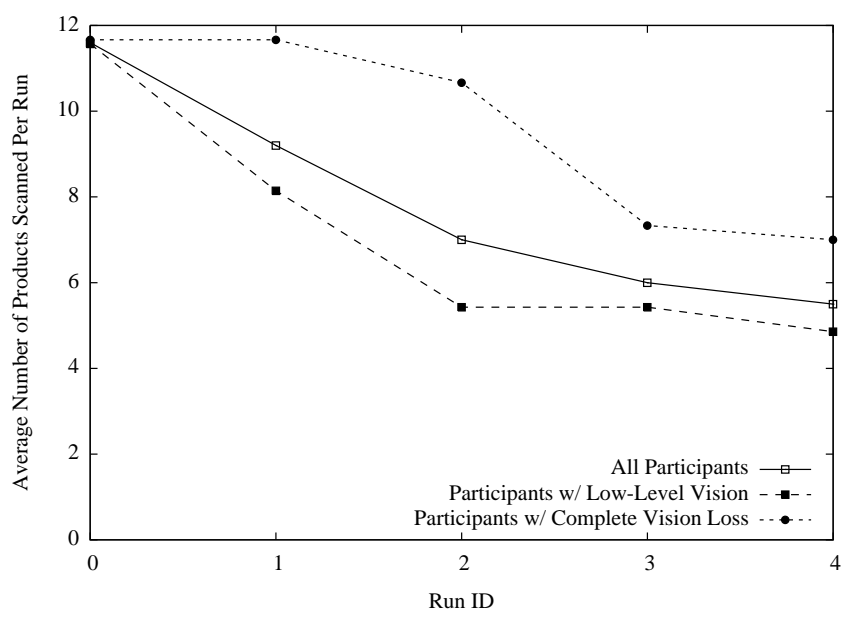

Fig. (9). Average number of items scanned over repeated runs. The baseline is not shown since products were located visually, not scanned, by the sighted guide in the baseline run. The ideal would be three products scanned.

The level of blindness, complete or low vision, caused a significant effect on the number of items scanned across all 5 runs, $\chi^{2}(1)=9.63, \mathrm{P}=0.0019$ with low vision shoppers scanning fewer numbers of items. The implication is that the partially sighted shoppers, since they have additional sensory information not available to the completely blind shoppers, are more efficient in their barcode scanning. Depending on the amount of partial vision and the type of visual impairment, some shoppers may not have to use the barcode scanner to find products in every situation. The difference in the rate at which the two groups improved over the five runs was not found to be significant.

\section{Qualitative Observations}

In addition to the statistical analysis reported, a number of qualitative observations were made regarding the participants, the way they used the device, and how they interacted with the store environment. In this section, we will discuss these observations and some of their implications.

\section{Shopping Techniques}

All participants were given instructions on how to use ShopTalk, yet several participants were observed using individual methods to enhance their search techniques. Two participants used touch to help locate their position relative to a product. Participant 1 noticed that marshmallows were located next to product 1 , and by the fourth run was locating the marshmallows with her hand and then moving from that position to the desired product. Participant 3 judged distances on her first run by running her finger along the shelf edges. There is a separator between shelf sections, which she counted in order to determine her position in the aisle.

Two other participants developed a different technique for finding product 1 . Product 1 was near the end of the first aisle, towards the back of the store in shelf section 18. Instead of beginning their search at shelf section 1 , they would walk to the opposite end of the aisle, shelf section 20 , and then backtracked to find shelf section 18 . 
Although participant 8 reported only being able to see light and dark, she was able to find product 1 on her first try with little apparent difficulty. During training, participants were told that a shelf section was approximately 1.22 meters wide. She reported taking advantage of this knowledge by counting her steps, allowing her to achieve a good level of accuracy without using the touch techniques used by others. She did use touch to help locate product 2, where she noticed that the shelf section next to product two was covered by a plastic divider which was different from the usual flat, metal shelves used in the majority of the store.

In each of these cases, the shoppers devised their own methods for accomplishing the task; none of these techniques was mentioned during training. ShopTalk provided them with enough flexibility that they could enhance their performance with a technique that worked for their navigation skill level and ability. One advantage of tracking these techniques is that over time, techniques seen to be the most useful could be explained to new users of the system, increasing the effectiveness of ShopTalk for all users.

\section{Environment}

Because the reported experiment was performed in a real-world supermarket, the store environment led to several observations. In general, other people, store employees and other shoppers, would move out of a participant's way if they noticed the participant. At times, though, other people did not notice the participant and the participant would have to adjust. For example, an elderly man walking slowly down an aisle slightly slowed the walking speed of one participant.

Another issue was that late evenings, when the experiments were performed, were also the time when employees restocked shelves. The employees would start placing large boxes in aisles and wheeling large carts with boxes around. The carts would stay in one spot for several minutes as the employees placed items on the shelves. If an employee noticed that a participant needed to pass the employee or to enter an aisle that a cart was blocking, the employee would move the cart and let the shopper pass. In several cases, however, the employee did not notice one participant. This participant, when repeatedly blocked from entering an aisle, went to the next aisle, proceeded to the back of the store, and returned to the desired aisle from the other end. Thus, the participant was using her own mental map and expectations of the store layout instead of following ShopTalk's instructions. In fact, ShopTalk's instructions never referred to the back of the store. All instructions regarding entering and exiting aisles were given solely in relation to the front of the store and aisles.

To show off items on sale, Lee's Marketplace sometimes stacks them in the aisles (see Fig. 10). The stacks and other promotional displays proved to be both advantageous and problematic. The third product in the experiment happened to be located two shelf sections after a large stack of cans of spaghetti sauce. Several of the participants were observed searching for this stack to help them locate the last item. The use of these stacks as landmarks is questionable over the long term since the items for sale change over time, some stacks gradually disappear and new ones appear at different locations. Stacks and other product displays also have to be negotiated carefully. Two participants accidentally hit a stack of spaghetti sauce cans, with each knocking a can of sauce to the floor. The end of the aisles where large displays of sales items are placed also provided similar challenges due to the differences of height of different displays. Participants would occasionally nudge items in these display areas as they walked by. Although we did not quantify the improvement at detecting stacks, we noticed that over repeated runs the participants were becoming better at avoiding product stacks.

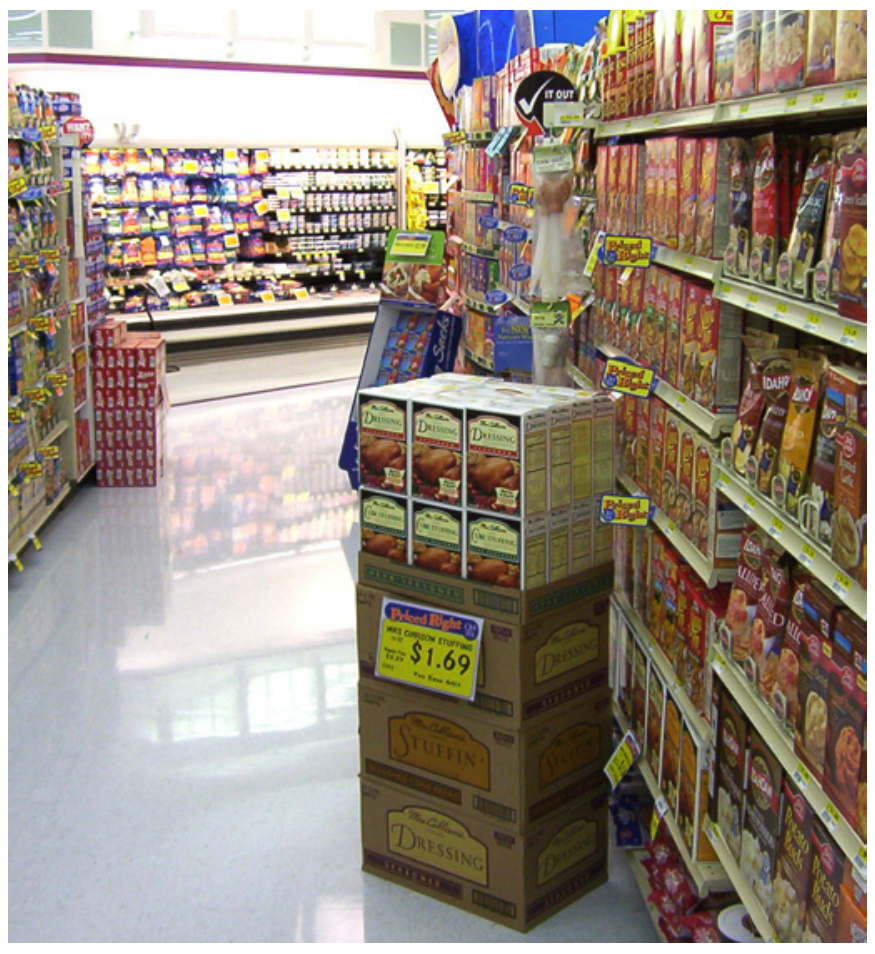

Fig. (10). Example of sale items stacked in the aisle.

\section{Ergonomics}

The experiment was designed to determine the feasibility of ShopTalk. However, the experiment also revealed some ergonomic issues with the devices. The largest issue brought up by four participants during informal discussions was there was a lot of equipment to manage. In addition to their usual cane or guide dog, the participant had to manage a barcode reader, a shopping basket, and press buttons on the keypad. One participant noted that in a real shopping situation she would also have to manage her preschool children. As noted previously, miniaturizing the hardware is our research priority.

The barcode scanner was typically, but not always, carried in the basket. When scanning for items, the guide dog handlers would instruct their guide dog to sit, lay their cane on the ground or change their grip on it in order to scan for items. The cane users would sometimes rest their cane on the shelves while scanning for barcodes. Several participants, however, seemed to be unaware of how their canes, dogs, and shopping baskets were extending beyond their personal space. For example, participant 4 held his cane and the shopping basket while he scanned barcodes on the shelves. Because he was concentrating on the scanning process, he did not seem to be aware of the fact that his cane and basket were hitting items on the shelf. While no items were knocked off the shelves, it is possible that an item could be 
knocked off a shelf. Two participants seemed unaware that products were stacked on one another. In these cases, they would pull out the bottom product although it would have been safer to take the top product.

\section{RELATED WORK}

This section briefly mentions several systems and commercial products that can aid shoppers with visual impairments. The technologies discussed here can be broken into two basic groups. The technologies discussed in the first section are general indoor navigation aids for the people with visual impairments. These technologies do not specifically target grocery shopping, but could be adapted to the problem of navigating in the locomotor space. The second section of related work discusses grocery-shopping aids.

\section{Indoor Localization and Navigation Aids}

Talking Lights [12] is a system that works with existing light fixtures. After special fixtures are installed, the light is modulated at different frequencies enabling the transmission of data which can be captured and decoded by optical receivers carried by a user. It has been used to encode route instructions to aid in wayfinding within buildings [17]. In a grocery store setting, this system could be used to guide a shopper to various sections of the store, e.g. produce, meat, dairy, as well as to individual aisles which would aid the shopper during the initial step of the product location task when they travel through locomotor space.

The Bat System [18] uses ultrasonic devices to provide localization and tracking services. Cricket [19] and Cicada [20], which are both localization systems, also use ultrasonic as well as radio frequency (RF). Drishti [21], an indoor and outdoor navigation system for the blind, integrated an ultrasonic localization system from the Australian company Hexamite [22]. In general, these systems require that a network or grid of transmitters be installed in the ceiling or on the walls. The user then carries a receiver that is used to triangulate its position. The Bat System uses time-of-flight of the ultrasonic signals, i.e., how long the signal takes to travel from the transmitter to the receiver, and is accurate to within $9 \mathrm{~cm}$ for $95 \%$ of its readings. Cricket also uses time-of-flight and has achieved an accuracy of $4 \times 4$ feet cells. Cicada is able to provide the locations within $5 \mathrm{~cm}$ average deviation.

Some systems use general radio signals while other systems take advantage of standard RF technologies such as Wi-Fi, Bluetooth, and ZigBee. The SpotON system [23] developed at the University of Washington uses tags that randomly transmit beacon signals. When other tags which detect a beacon they use the received signal strength information (RSSI) to estimate distance to the transmitter.

The RADAR system [24] from Microsoft Research is designed to locate and track users inside buildings. It processes the Wi-Fi signal strengths received by a standard network cards received from 802.11 base stations using a signal propagation modeling method to calculate the mobile users' positions and is capable of achieving a median error distance of 2 to 3 meters. In the Place Lab [25] based system described in [26], signals from cell phone networks, Wi-Fi signals, and data from an accelerometer are fused to provide location information with an average accuracy of 20 to 30 meters. Using Wi-Fi, fingerprinting systems like RADAR can improve the resolution to 1 to 2 meters. Commercial products now exist that provide $\mathrm{Wi}$-Fi based localization services. For example, the Ekahau RTLS (Real Time Location System) sold by Ekahau [27] also uses Wi-Fi with the company reporting an expected accuracy of 1 to 3 meters. Their system collects RSSI values from Ekahau tags worn by users which are processed by a server to determine the tags' locations.

Similar to the Wi-Fi systems are those based on Bluetooth. The system described in [28] uses a Bayesian based approach on the RSSI received from Bluetooth dongles. Essentially a variation on the triangulation approach, it achieved an accuracy of 2 meters with a standard deviation of 1.2 meters when using three service points. One of the newest wireless standards to be exploited for localization is the ZigBee standard [29]. In [30], the RSSI from nodes on a ZigBee based network is used for localization achieving an accuracy of 1.5 to 2 meters.

In RFID systems, RFID tags are placed at strategic locations and the user carries an RFID reader, or small RFID readers are placed in the area for localization and the user carries an RFID tag. Whenever an antenna detects a tag, the mobile user's location is determined to be within some distance of the fixed location. LANDMARC [13] uses stationary RFID readers. Stationary reference RFID tags are placed around the building, providing a means of location calibration. Location is calculated using a mapping from power levels of received signals to RSSI for both the reference tags and the tags on the mobile users. In experiments, four readers and one reference tag per square meter located objects within 1 to 2 meters.

One disadvantage of these systems is the overhead required to manage these systems and instrument the environment. Tags and beacons need to be installed at the best locations, and, depending on the system, may be perceived as negatively affecting the appearance of the store. Several systems require multiple devices needing power. If battery powered, devices need to be monitored on a periodic basis. If non-battery powered, extra cabling will have to be installed. Additionally, calibration may be required for most of these systems in order to achieve the highest accuracy.

\section{Grocery Shopping Aids}

For shoppers who have a low-level visual impairment but still have a relatively high level of sight, a hand-held magnifying glass is a low-cost, low-tech tool which can be useful in a shopping situation. There are also electronic magnifiers on the market, such as Looky [31] and OPAL [32], which have advantages over the hand-held magnifying such as zoom and image enhancement. The disadvantage of the electronic magnifiers is that they require power and are significantly more expensive than a magnifying glass.

For many shoppers with visual impairments, magnifiers may not meet their needs. Various systems have been developed to identify products. Trinetra [33] uses a smartphone and a Bluetooth barcode scanner to identify products. Scanned items are looked up in a database. The system does not address the shopper's performance in the locomotor and search spaces. The implicit assumption is that the shopper is sufficiently familiar with the store to locate target products. 
This assumption, while valid for some small convenience stores, does not hold in large supermarkets.

The i.d. mate OMNI [34], a commercial product from En-Vision America, is a standalone, talking barcode scanner. It has an on-board database of 1.6 million UPC barcodes and includes extra data such as ingredients and nutrition information for some products. It also allows the user to add new barcodes not found in the current database. Grozi [35] is a system that uses image recognition to identify individual products. 676 images of the product captured under ideal situations and 11,194 images image found on the web were used to represent 120 products. The ideal images are then used as training data and the web images were used as the test data. The SIFT algorithm [36] is then used to identify products form images captured by a camera on a mobile device.

These systems are aimed at product identification, not product search and retrieval. If the shopper is able to find the exact location of the target product, then these systems may help to ensure that the product picked up is the correct product. On the other hand, if any amount of search is involved in the haptic space, the use of these systems could become tedious, since no product maps are provided and no guidance is given in the locomotor, search, and haptic spaces. If the shopper needs any sort of assistance to navigate through the locomotor or search spaces, then these tools are insufficient to provide independence in blind grocery shopping.

\section{DISCUSSION}

ShopTalk is a proof-of-concept prototype. There remain many avenues for future work. Our most immediate objective is to migrate the system to a more conventional and mobile platform, such as a cell phone or a PDA. Cell phones and PDAs now have the ability to perform text-to-speech (TTS), contain moderately large amounts of storage space, and can connect wirelessly to other devices using Bluetooth. However, in the final analysis, the actual hardware realization is of secondary importance, because ShopTalk is a software system that can be realized on different hardware platforms.

ShopTalk's BCM, the data structure for storing topological information about barcodes on shelves, was manually built, because we were not granted access to the inventory management system of Lee's Marketplace. In a production version, the system would connect to the store's inventory control database and look up pertinent product information. Inventory control databases would make it possible to deliver dynamic information about prices, ingredient lists, nutrition facts, and items on sale. A production version would also provide sort type of product identification, which is currently not supported since ShopTalk is still at the early prototype stage.

Several participants, including one who was completely blind, mentioned that when shopping, they use the regular, wheeled shopping carts instead of the hand baskets used during the experiment. Technically speaking, the manner in which a shopper carries products is not central to ShopTalk. ShopTalk will be useful to a shopper with a visual impairment who wants to use a shopping cart if the shopper can independently manage the shopping cart while navigating the store. Since ShopTalk is a software guidance system, there is no limit on the number of items the shopper could shop for during one shopping session. As long as the shopper is capable of navigating the store with ShopTalk's verbal route instructions while handling a cart full of products, the shopper will be able to shop for large numbers of products. This, however, is a hypothesis to be verified in the future.

The scope of the experiment limited the participants to only shopping for products in aisles with shelves. Other areas in the store have their products arranged differently or present different challenges than the aisles with shelves. These areas include the freezer sections with doors, the open refrigerator areas, such as dairy and meat sections, delis, bakeries, and produce sections that are sometimes less structured than aisles. In the end, ShopTalk may not make all areas of a grocery store completely accessible to shoppers with visual impairments. However, we are reasonably certain that ShopTalk helps many shoppers achieve a higher level of shopping independence than what is currently available.

Currently, ShopTalk does not handle product identification so it is possible that a shopper could be at the correct location for a target product and yet retrieve an incorrect product. Product identification for ShopTalk will be addressed in future work, but we do not see it as a major obstacle. Well-managed stores maintain their shelves in order because their business depends on it, and employees periodically inspect the shelves to remove misplaced items and restock the sold items. In time, the shopper will learn valid haptic cues to identify correct products. For example, a peanut butter jar can be easily distinguished from a can of corn by touch. In the case of identical containers, either a second scan of the barcode on the product's label or verification at checkout could be used to resolve product questions. Finally, some shoppers with partial vision will be able to identify the products visually. The problem of individual product identification can most likely be solved with a combination of technical solutions, e.g. computer vision, and non-technical solutions that rely partly on the shopper's intelligence and ability to adapt and partly on the willingness of the store to keep their customers satisfied.

It is unlikely that ShopTalk will be of use to all people with visual impairments. The system is designed for mobile individuals who have sufficient O\&M skills to navigate indoor environments independently and have no other impairments that could potentially impede navigation, such as serious cognitive or physical disabilities. ShopTalk is not designed to address physical limitations that may prevent individuals from grocery shopping, such as not being able to reach high or low shelves or being unable to carry heavy items. The level of visual impairment is not a factor in that ShopTalk can be used by people with complete or partial vision loss.

We are hesitant to generalize our findings across the entire population of those with visual impairments due to our small sample size. However, we are reasonably certain that we have shown that a device, such as ShopTalk, is feasible, because all our participants, even those who were completely blind, successfully found the target products. Our experiments have shown that the utility of the device improves with familiarity and repetition. This effect is consistent, and does not appear to depend on the level of blindness or any other sample characteristics that we measured. The experi- 
ments show that, with repetition, the shopping task is performed more efficiently, allowing the shopper to shop at a rate which, in some cases, is comparable to a shopper guided by a sighted aid.

\section{CONCLUSION}

ShopTalk takes advantage of both the navigation skills that people use in their everyday lives and the inventory infrastructure already present in many grocery stores. To use the system, individuals with visual impairments do not have to master a new set of navigation skills. Shoppers may continue to use their navigation aid of choice in the manner to which they are accustomed. When the shopper is moving about in the locomotor space, the system is unobtrusive and gives commands only if prompted. If the shopper already knows the general store layout, no commands are given by the system until a barcode is scanned. Thus, shoppers do not have to contend with constant sound from the system while trying to pay attention to the environment for audio cues.

Using the store's existing infrastructure simplifies installation. No extra hardware needs to be installed or maintained, which has the added benefit of not affecting the appearance of the store. The shelf labels provide extremely accurate positioning, assisting with error correction in the locomotor space, and allowing the shoppers to perform barcode scans that guide them through the search and haptic spaces ultimately allowing the shopper to find the location of specific products out of thousands.

ShopTalk represents an important step in providing a tool that will allow people with visual impairments to retain their independence and perform grocery shopping, a task that many sighted people take for granted. We recognize that since the number of participants in our experiments was small, our quantitative findings should be interpreted with caution. Nevertheless, our findings suggest that verbal route instructions and barcode scans may be sufficient for enabling some visually impaired shoppers to shop for shelved products in modern supermarkets independently.

\section{ACKNOWLEDGEMENTS}

We would like to thank Mr. Sachin Pavithran, an assistive technology specialist at the Utah State University Center for Persons with Disabilities, for helping us organize the focus group discussion on independent blind shopping. The second author would like to acknowledge that this research has been supported, in part, through NSF grant (IIS0346880), the National Eye Institute of the National Institutes of Health under Grant (1 R41 EY017516-01A1), and three Community University Research Initiative (CURI) grants (CURI-04, URI-05, and CURI-06) from the State of Utah.

\section{REFERENCES}

[1] Passini R, Proulx G. Wayfinding without vision: an experiment with congenitally totally blind people. Environ Behav 1988; 20(2): 227-52.

[2] Food Marketing Institute Research. The Food Retailing Industry Speaks 2006. Food Marketing Institute 2006.

[3] Kulyukin V, Gharpure C, Nicholson J. RoboCart: toward robotassisted navigation of grocery stores by the visually impaired. Intelligent Robots and Systems, 2005 (IROS 2005) 2005 IEEE/RSJ International Conference on. 2-6 Aug 2005; pp. 2845-50.
[4] Burrell A. Robot lends a seeing eye for blind shoppers. USA Today 2005; July 11: 7D.

[5] Peapod [Homepage on the Internet]; c1990 - 2008 [cited 2008 November 1]. Available from: http://www.peapod.com/

[6] Kulyukin V, Gharpure C, Pentico C. Robots as interfaces to haptic and locomotor spaces. In: HRI '07: Proceeding of the ACM/IEEE international conference on Human-robot interaction. New York, NY, USA: ACM 2007. pp. 325-31.

[7] Mcquistion L. Ergonomics for one. Ergonomics in design: the quarterly of human factors applications. Hum Factors Ergon Soc 1993; 1(1): 9-10.

[8] Kulyukin VA, Gharpure C. Ergonomics for- one in a robotic shopping cart for the blind. In: HRI '06: Proceeding of the 1st ACM SIGCHI/SIGART conference on Human-robot interaction. New York, NY, USA: ACM 2006; pp. 142-9.

[9] Gharpure C, Kulyukin V. Robot-assisted shopping for the blind: issues in spatial cognition and product selection. Int J Serv Rob 2008; 1(3): 237-51.

[10] Tversky B, Morrison JB, Franklin N, Bryant DJ. Three spaces of spatial cognition. Prof Geogr 1999; 51(4): 516-24.

[11] Freundschuh S, Egenhofer M. Human conceptions of spaces: implications for geographic information systems. Trans GIS 1997; 2(4): 361-75.

[12] Talking Lights LLC [Homepage on the Internet]; 2008 [cited 2008 November 1]. Available from: http://www.talking-lights.com.

[13] Ni LM, Liu Y, Lau YC, Patil AP. LANDMARC: indoor location sensing using active RFID. Wirel Netw 2004; 10(6): 701-10.

[14] Gaunet F, Briffault X. Exploring the functional specifications of a localized wayfinding verbal aid for blind pedestrians: simple and structured urban areas. Hum-Comput Interact 2005; 20(3): 267314.

[15] Kulyukin V, Nicholson J, Ross D, Marston J, Gaunet F. The blind leading the blind: toward collaborative online route information management by individuals with visual impairments. Papers from the AAAI Spring Symposium 2008.

[16] Nicholson J, Kulyukin V. ShopTalk: Independent blind shopping = verbal route directions + barcode scans. In: Proceedings of the 30th Annual Conference of the Rehabilitation Engineering and Assistive Technology Society of North America (RESNA 2007). Phoenix, AZ 2007.

[17] Leeb SB, Hovorka GB, Lupton EC, et al. Assistive communication systems for disabled individuals using visible lighting. In: 15th International Technology and Persons with Disabilities Conference. Northridge, CA 2000.

[18] Harter A, Hopper A, Steggles P, Ward A, Webster P. The anatomy of a contextaware application. Wirels Netw 2002; 8(2/3): 187-97.

[19] Priyantha NB, Chakraborty A, Balakrishnan H. The cricket location-support system. In: MobiCom '00: Proceedings of the 6th annual international conference on Mobile computing and networking. New York, NY, USA: ACM 2000; pp. 32-43.

[20] Jiang W, Chen Y, Shi Y, Sun Y. The design and implementation of the Cicada wireless sensor network indoor localization system. In: ICAT Workshops. IEEE Computer Society 2006; pp. 536-41.

[21] Ran L, Helal S, Moore S. Drishti: An Integrated Indoor/Outdoor Blind Navigation System and Service. In: PERCOM '04: Proceedings of the Second IEEE International Conference on Pervasive Computing and Communications (PerCom'04). Washington, DC, USA: IEEE Computer Society 2004. p. 23.

[22] Hexamite [Homepage on the Internet]; 2008 [cited 2008 November 1]. Available from: http://www.hexamite.com.

[23] Hightower J, Vakili C, Borriello G, Want R. Design and calibration of the SpotON Ad-Hoc location sensing system; 2001. Unpublished. Available from: http://seattle.intel-research.net/people/jhigh tower/pubs/hightower2000indoor/ hightower2000indoor.pdf.

[24] Bahl P, Padmanabhan VN. RADAR: an inbuilding RF-based user location and tracking system. INFOCOM 2000 Nineteenth Annual Joint Conference of the IEEE Computer and Communications Societies Proceedings IEEE 2000; 2: 775-84.

[25] LaMarca A, Chawathe Y, Consolvo S, et al. Place lab: device positioning using radio beacons in the wild. In: Proceedings of the Third International Conference on Pervasive Computing. Lecture Notes in Computer Science. Springer-Verlag 2005; pp. 116-33.

[26] Welbourne E, Lester J, LaMarca A, Borriello G. Mobile context inference using low-cost sensors. In: Strang T, Linnhoff-Popien C, Eds. LoCA. vol. 3479 of Lecture Notes in Computer Science. Springer 2005; pp. 254-263. 
[27] Ekahau [Homepage on the Internet]; c2000-2008 [cited 2008 November 1]. Available from: http://www.ekahau.com.

[28] Wendlandt K, Berbig M, Robertson P. Indoor localization with probability density functions based on Bluetooth. Personal, Indoor and Mobile Radio Communications, 2005 PIMRC 2005 IEEE 16th International Symposium on 2005; 3: 2040-44.

[29] ZigBee Alliance [Homepage on the Internet]; c2007-2008 [cited 2008 November 1]. Available from: http://www.zigbee.org.

[30] Sugano M, Kawazoe T, Ohta Y, Murata M. Indoor localization system using RSSI measurement of wireless sensor network based on ZigBee standard. In: Fapojuwo AO, Kaminska B, Eds. Wireless and optical communications. IASTED/ACTA Press 2006.

[31] Steller Technology. Looky handheld magnifier [Product page on the Integet]; 2008 [cited 2008 November 1]. Available from: http://www.steller-technology.co.uk/looky.php.
[32]

Freedom Scientific. OPAL Ultra-portable video magnifier [Product page on the Integet]; 2008 [cited 2008 November 1]. Available from: http://www.freedomscientific. com/products/lv/opal-productpage.asp.

[33] Lanigan PE, Paulos AM, Williams AW, Rossi D, Narasimhan P. Trinetra: Assistive Technologies for Grocery Shopping for the Blind. In: International IEEE-BAIS Symposium on Research on Assistive Technologies (RAT '07). Dayton, OH 2007.

[34] i.d. mate OMNI [Product page on the internet]. En-Vision America; c2007-2008 [cited 2008 November 1]. Available from: http://www.envisionamerica.com/idmate/

[35] Merler M, Galleguillos C, Belongie S. Recognizing groceries in situ using in vitro Training Data. 2007 IEEE Conference on Computer Vision and Pattern Recognition 2007 June, pp. 1-8.

[36] Lowe DG. Distinctive image features from scale-invariant keypoints. Int J Comput Vis 2004; 60(2): 91-110.

(C) Nicholson et al.; Licensee Bentham Open.

This is an open access article licensed under the terms of the Creative Commons Attribution Non-Commercial License (http: //creativecommons.org/licenses/by$\mathrm{nc} / 3.0 /$ ) which permits unrestricted, non-commercial use, distribution and reproduction in any medium, provided the work is properly cited. 